\title{
Safety and efficacy of aneurysm treatment with WEB: results of the WEBCAST study
}

\author{
Laurent Pierot, MD, PhD, ${ }^{1}$ Vincent Costalat, MD, PhD, ${ }^{2}$ Jacques Moret, MD, PhD, ${ }^{3}$ \\ Istvan Szikora, MD, ${ }^{4}$ Joachim Klisch, MD, ${ }^{5}$ Denis Herbreteau, MD, PhD, ${ }^{6}$ \\ Markus Holtmannspötter, MD, ${ }^{7}$ Werner Weber, MD, ${ }^{8}$ Anne-Christine Januel, MD, ${ }^{9}$ \\ Thomas Liebig, MD, PhD, ${ }^{10}$ Vojtech Sychra, MD, ${ }^{5}$ Christoph Strasilla, MD, ${ }^{5}$ \\ Christophe Cognard, MD, PhD, ${ }^{9}$ Alain Bonafé, MD, PhD, ${ }^{2}$ Andrew Molyneux, MD, PhD, ${ }^{11}$ \\ James V. Byrne, MD, ${ }^{11}$ and Laurent Spelle, MD, PhD ${ }^{3}$
}

1Department of Neuroradiology, Hôpital Maison-Blanche, Université Reims-Champagne-Ardenne, Reims; ${ }^{2}$ Department of Neuroradiology, CHU Montpellier; ' ${ }^{3}$ Department of Neuroradiology, Hôpital Beaujon, Assistance Publique-Hôpitaux de Paris, Clichy; ${ }^{6}$ Department of Neuroradiology CHU Tours; ${ }^{9}$ Department of Neuroradiology CHU Toulouse, France; ${ }^{4}$ Department of Neuroradiology, National Institute of Neurosciences, Budapest, Hungary; ${ }^{5}$ Department of Neuroradiology, Helios General Hospital, Erfurt; ${ }^{8}$ Department of Neuroradiology, Knappschaftskrankenhaus, Recklinghausen; ${ }^{10}$ Department of Neuroradiology Universitätsklinikum Köln, Cologne, Germany; ${ }^{7}$ Department of Neuroradiology, Rigshospitalet, Copenhagen, Denmark; and ${ }^{11}$ Oxford Neurovascular \& Neuroradiology Research Unit, Oxford Radcliffe Hospital, Oxford, United Kingdom

OBJECTIVE WEB is an innovative intrasaccular treatment for intracranial aneurysms. Preliminary series have shown good safety and efficacy. The WEB Clinical Assessment of Intrasaccular Aneurysm Therapy (WEBCAST) trial is a prospective European trial evaluating the safety and efficacy of WEB in wide-neck bifurcation aneurysms.

METHODS Patients with wide-neck bifurcation aneurysms for which WEB treatment was indicated were included in this multicenter good clinical practices study. Clinical data including adverse events and clinical status at 1 and 6 months were collected and independently analyzed by a medical monitor. Six-month follow-up digital subtraction angiography was also performed and independently analyzed by a core laboratory. Success was defined at 6 months as complete occlusion or stable neck remnant, no worsening in angiographic appearance from postprocedure, and no retreatment performed or planned.

RESULTS Ten European neurointerventional centers enrolled 51 patients with 51 aneurysms. Treatment with WEB was achieved in 48 of 51 aneurysms (94.1\%). Adjunctive implants (coils/stents) were used in 4 of 48 aneurysms (8.3\%). Thromboembolic events were observed in 9 of 51 patients (17.6\%), resulting in a permanent deficit (modified Rankin Scale [mRS] Score 1) in 1 patient (2.0\%). Intraoperative rupture was not observed. Morbidity (mRS score > 2) and mortality were $2.0 \%$ ( 1 of 51 patients, related to rupture status on entry to study) and $0.0 \%$ at 1 month, respectively. Success was achieved at 6 months in $85.4 \%$ of patients treated with WEB: 23 of 41 patients (56.1\%) had complete occlusion, 12 of $41(29.3 \%)$ had a neck remnant, and 6 of 41 (14.6\%) had an aneurysm remnant.

CONCLUSIONS The WEBCAST study showed good procedural and short-term safety of aneurysm treatment with WEB and good 6-month anatomical results.

Clinical trial registration no.: NCT01778322 (clinicaltrials.gov)

http://thejns.org/doi/abs/10.3171/2015.2.JNS142634

KEY WORDS intracranial aneurysms; endovascular treatment; WEB; flow-diverter device; vascular disorders

$\mathrm{E}$ NDOVASCULAR treatment is now the first-line treatment for both ruptured and unruptured aneurysms.1,7,17 However, aneurysms with a complex anatomy (singularly wide-neck aneurysms) are in some cases untreatable or difficult to treat with standard coiling. For this reason, more complex endovascular techniques have been developed, such as balloon-assisted coiling, stent-assisted coiling, and flow diversion. ${ }^{18}$

Flow disruption is a new endovascular approach, which involves placement of an endosaccular device (WEB), 
which modifies the blood flow at the level of the neck and induces intraaneurysmal thrombosis. The WEB was designed to treat wide-neck bifurcation aneurysms. ${ }^{5,6,9,15,16}$ The device has been progressively developed from a duallayer version (WEB DL) to single-layer versions (WEB SL and WEB SLS [single layer spherical]) (see WEB Devices). This treatment was evaluated previously in retrospective series and showed good safety results. ${ }^{5,6,9,15,16}$

WEBCAST (WEB Clinical Assessment of Intrasaccular Aneurysm Therapy) is a prospective, observational, multicenter, controlled, European study with 2 major objectives: 1) to carefully evaluate the safety of this treatment with an independent evaluation of all adverse events and morbidity/mortality; and 2) to evaluate its efficacy. This is the first multicenter study to prospectively assess safety and efficacy of the WEB.

\section{Methods}

The study received national regulatory authorization (CCTIRS: Consultative Committee of Information Processing in Healthcare Research program), Reims Institutional Review Board approval and CNIL (National Commission for Data Processing and Freedom) approval, and ethics committee approval. Written informed consent was obtained for all patients. Two patients withdrew consent: one after the procedure and the other between 1 and 6 months.

\section{Trial Design}

WEBCAST is a single-arm, prospective, consecutive, multicenter, European study dedicated to the evaluation of WEB treatment for wide-neck bifurcation aneurysms (clinical trial registration no. NCT01778322 [clinicaltrials. gov]).

Inclusion criteria were ruptured and unruptured bifurcation aneurysms located at the basilar artery (BA), middle cerebral artery (MCA), anterior communicating artery (ACoA), and internal carotid artery (ICA) terminus, with a size compatible with WEB treatment and a wide neck $(\geq$ $4 \mathrm{~mm}$ ). Subjects had to have been between 18 and 75 years of age and harboring a single aneurysm requiring treatment. Patients with previously treated aneurysms were excluded, as were patients with more than 1 aneurysm requiring treatment within 30 days. Patient follow-up is required at 30 days (safety), 6 months (safety plus imaging), 12 months (safety plus imaging), and annually to 5 years.

Per protocol, primary analysis was conducted at 6 months. Safety parameters included incidence of neurological complications occurring during the WEB procedure, device- and procedure-related stroke within 30 days of the WEB procedure, device- and procedure-related death within 30 days of the WEB procedure, rehemorrhage rates (for ruptured aneurysms), new hemorrhage rates (for unruptured aneurysms), retreatment rates, and clinical status at 6 months assessed using the modified Rankin Scale (mRS).

Primary analysis was conducted at 6 months of followup, defined as durability of occlusion compared with postprocedure angiogram as determined by Core Lab.

Efficacy (durability) was defined as meeting all of the following criteria: complete angiographic occlusion or stable neck remnant at 6 months (adequate occlusion), with occlusion stable or improved from baseline, and no retreatment performed or planned (see Statistical Analysis).

\section{WEB Devices}

WEB devices are intrasaccular, detachable, nitinol cages that are placed in the aneurysm to disrupt the flow at the level of the neck and create aneurysmal thrombosis. The first device available for clinical use was the WEBDL (dual layer), which was composed of 2 layers. WEBSL (single layer) and WEB-SLS (single layer spherical) became available secondarily. Patients included in WEBCAST were treated using the WEB-DL device.

\section{Procedural Modalities}

The treatment of aneurysms with WEB was done using similar techniques as are used in the treatment of aneurysms with coils (e.g., general anesthesia, intraoperative treatment with intravenous heparin, and single or double femoral approach). Preoperative, intraoperative, and postoperative antiplatelet therapy was managed autonomously at each center.

After accurate evaluation of aneurysm anatomy (aneurysm morphology, aneurysm transverse diameter and height, and neck size) by the treating physician using digital subtraction angiography (DSA), it was determined whether treatment with WEB was indicated and feasible without the use of adjunctive devices, and appropriate device sizing was determined.

The treatment was usually performed using triaxial access (long introducer sheath/distal access catheter/microcatheter). After the positioning of a distal access catheter into the ICA or vertebral artery, the aneurysm was catheterized using a microcatheter, and the WEB size was chosen according to aneurysm measurements and positioned in the aneurysmal sac per methods described by Pierot et al. ${ }^{16}$ If the treatment plan included planned use of ancillary implant devices, the patient was not enrolled in the study. Treatment with ancillary devices (coils, stents, or flow diverters) was allowed to be used during the procedure if deemed necessary by the physician, and was not considered a treatment failure.

\section{Data Collection}

Each center completed a patient file with the following data: patient's age and sex; aneurysm status; aneurysm characteristics including location, size, and neck size; date of the procedure; perioperative antiplatelet medications; occurrence of adverse events during or after the procedure; and use of additional devices during the procedure (coils, remodeling balloons, stents, or flow diverters). All adverse events were collected in this good clinical practices series even if no specific treatment was needed or if no clinical worsening was observed. Thromboembolic events/appearance of thrombus were diagnosed intraoperatively by angiography regardless of type (clotting near the neck of the aneurysm, clotting in the distal branches, and parent vessel occlusion). Postoperative thromboembolic events were diagnosed by MRI and/or DSA performed in 
cases of sudden neurological compromise. Intraoperative rupture was diagnosed by the exit of the tip of the coil or the microcatheter outside the limit of the aneurysmal sac and/or extravasation of contrast medium. Adverse events were reported even if no clinical modification was associated with them.

The preoperative Hunt and Hess grade was collected in cases of ruptured aneurysms. The mRS score was evaluated by a physician not directly involved in the treatment before treatment (unruptured aneurysms) or before bleeding on the basis of patient and relative feedback (ruptured aneurysms) at 1 month and at 6 months.

For aneurysms treated with WEB, preoperative, postoperative, and midterm (6 months) DSA images were collected and evaluated by an independent core laboratory. For DSA, frontal, lateral, and working views were collected as well as 3D angiography when available.

\section{Data Analysis}

Clinical data were monitored and analyzed including all adverse events by an independent medical monitor (A.M.). Morbidity was defined as an $\mathrm{mRS}$ score $>2$ when the preoperative $\mathrm{mRS}$ score was $\leq 2$ (or in case of ruptured aneurysms), and any increase of 1 point when preoperative mRS score was $>2$.

An independent Core Lab (J.V.B.) analyzed the images (pre- and postoperative and 6-month follow-up) using a validated 3-grade scale: complete occlusion (including opacification of the proximal recess), neck remnant, and aneurysm remnant. ${ }^{3,5}$

\section{Statistical Analysis}

Continuous variables were described as mean $\pm \mathrm{SD}$, and nonnormally distributed variables were described as median and interquartile range. Analyses were conducted using statistical software (version 8, StaXact).

A performance goal was developed based on review of the literature as well as the success rate from the Cerecyte Coil Trial (CCT) of 245 of $433(56.6 \%)$ for all aneurysm types and a region of noninferiority of $15 \%$ (performance goal $=41.6 \%) .{ }^{8}$ With a proposed WEB success rate of $62 \%$, $80 \%$ power, and 1 -sided $\alpha$ of 0.05 , the required sample size for analysis was 39 subjects with completed 6-month follow-up. The null (H0) and alternative (Ha) hypotheses for this end point were: $\mathrm{H}_{0}: \mathrm{P}_{\mathrm{WEB}} \leq 0.4158$ versus $\mathrm{H}_{\mathrm{a}}$ : $\mathrm{P}_{\mathrm{WEB}}$ $>0.4158$, where $P_{\text {WEB }}$ is the success rate at 6 months in the treated population. Thus, to achieve the efficacy end point, the efficacy rate must be statistically significantly higher than the performance goal.

\section{Results}

\section{Patient and Aneurysm Population}

Between December 2011 and February 2014, 10 European centers included 51 patients ( 35 females) ranging in age from 33 to 74 years (mean $55.6 \pm 10.8$ years) with 51 aneurysms.

Three (5.9\%) aneurysms were ruptured and 48 (94.1\%) were unruptured. Clinical status is summarized in Table 1. Twenty-nine aneurysms were located in the MCA (56.9\%), 12 in the BA (23.5\%), 6 in the ICA terminus (11.8\%), and
TABLE 1. Baseline Hunt and Hess (ruptured) and mRS (unruptured)

\begin{tabular}{cc}
\hline Baseline Assessment & ITT No. of Patients \\
\hline Hunt \& Hess grade (ruptured) & $3(5.9 \%)$ \\
\hline I & 0 \\
\hline II & 2 \\
\hline III & 1 \\
\hline mRS score (unruptured) & $48(94.1 \%)$ \\
\hline 0 & 41 \\
\hline 1 & 6 \\
\hline 2 & 1 \\
\hline
\end{tabular}

4 in the ACoA (7.8\%). Aneurysm neck size measured during the procedure was $\geq 4 \mathrm{~mm}$ in all aneurysms, and the mean neck size was $5.6 \mathrm{~mm}$. Forty-one aneurysms had a maximum size $<10 \mathrm{~mm}(80.4 \%)$, and the mean aneurysm diameter was $8.2 \mathrm{~mm}$ per Core Lab.

\section{Perioperative Antiplatelet Medications}

Before the procedure, 21 patients had no antiplatelet treatment, 16 had single antiplatelet medication, and 14 had 2 antiplatelet medications. During the procedure 12 patients had no antiplatelet treatment, 26 had single antiplatelet medication, and 13 had 2 antiplatelet medications. At discharge, 8 patients were receiving no antiplatelet treatment; 32, single antiplatelet medication; and 11, 2 antiplatelet medications. At 1 month (48 patients treated with WEB), 20 patients were receiving no antiplatelet treatment; 19, single antiplatelet medication; and 9, 2 antiplatelet medications. At 6 months (46 alive patients, see Mortality/Morbidity at 1 and 6 Months), 25 patients were receiving no antiplatelet treatment; 18 , single antiplatelet medication; and 3, 2 antiplatelet medications.

Table 2 examines the relationship between thromboembolism and antiplatelet treatment before and/or during the procedure. A total of 9 thromboembolic events occurred during the study. For patients receiving antiplatelet prophylaxis before and/or during the procedure, 8 thromboembolic events occurred in 39 patients (20.5\%). Only 1 thromboembolic event $(8.3 \%)$ occurred in 12 patients receiving no antiplatelet prophylaxis before and/or during the procedure. Of the 8 thromboembolic events occurring in patients receiving prophylaxis, 6 thromboembolic events occurred $(23.1 \%)$ in the 26 patients on single antiplatelet treatment and 2 thromboembolic events occurred $(15.4 \%)$ in the 13 receiving dual antiplatelet treatment. There was no statistically significant difference between the use of antiplatelet prophylaxis before and/or during the procedure and no use of antiplatelet prophylaxis ( $\mathrm{p}$ $=0.6663$ ) for thromboembolic events. There was also no statistically significant difference between single and dual antiplatelet prophylaxis for thromboembolic event $(\mathrm{p}=$ 0.6942) (Table 2).

\section{Treatment Feasibility and Adjunctive Treatments}

Treatment was successfully performed with WEB in all but 3 patients (94.1\%). In 2 patients (one with an MCA 
TABLE 2. Occurrence of events in relation with antiplatelet treatment

\begin{tabular}{|c|c|c|c|c|}
\hline Preop \&/or Intraop Regimen & Total No. of Patients & TE Event & No TE Event & p Value $(99 \% \text { confidence level })^{*}$ \\
\hline No. of patients & 51 & 9 & 42 & \\
\hline No antiplatelet prophylaxis & 12 & 1 & 11 & \multirow{2}{*}{$p=0.6663 \dagger$} \\
\hline Any antiplatelet prophylaxis & 39 & 8 & 31 & \\
\hline Single antiplatelet prophylaxis $\ddagger$ & 26 & 6 & 20 & \multirow{2}{*}{$p=0.6942 \S$} \\
\hline Dual antiplatelet prophylaxis & 13 & 2 & 11 & \\
\hline $\begin{array}{l}\text { TE = thromboembolic. } \\
* \text { One-sided Fisher exact test to a signi } \\
\dagger p \text { value comparing the occurrence of } \\
\ddagger \text { Aspirin or clopidogrel. Only } 2 \text { patient } \\
\S p \text { value comparing the occurrence of }\end{array}$ & $\begin{array}{l}\text { ance level of } 0.01 \text {. } \\
\text { with no antiplatelet to } \\
\text { ok clopidogrel at or aft } \\
\text { with single therapy to }\end{array}$ & $\begin{array}{l}\text { occurrence } \\
\text { רe index pr } \\
\text { occurrence }\end{array}$ & $\begin{array}{l}\text { E with antiplatel } \\
\text { ure. } \\
\text { E with dual ther }\end{array}$ & rapy. \\
\hline
\end{tabular}

aneurysm and the other with an ICA terminus aneurysm), the WEB was deployed but was protruding in the bifurcation, leading to its withdrawal. In 1 patient (BA aneurysm), the appropriate device sizing was not available for the treatment. Two of these 3 patients were treated with coils; the third patient was treated with coils and a stent.

Among patients treated with WEB, adjunctive implants were used in 4 of 48 aneurysms (8.3\%). Coils were used in 3 patients, in which aneurysm occlusion was incomplete after WEB delivery. In 1 patient, a stent was used to reduce WEB protrusion.

\section{Technical Problems and Adverse Events}

Technical problems were encountered in 8 patients $(15.7 \%)$, including prolonged detachment $(n=3)$ and WEB protrusion $(n=5)$. These events were clinically asymptomatic. There were 2 serious adverse events adjudicated as device related: postprocedure transient ischemic attack (recovered without sequelae) and immediate postprocedural stroke with hemiparesis and aphasia. Overall, thromboembolic events/appearance of thrombus were reported in 9 of 51 patients $(17.7 \%$ ) with appearance of thrombus/no deficit in $6(11.7 \%)$, transient symptoms with no sequelae in $2(3.9 \%)$, and permanent deficit in $1(2.0 \%)$. This latter patient (mentioned above) had postprocedural hemiparesis and aphasia and had an mRS score of 1 (reduction of fine motor skill with the right hand and, when tired, feeling slow with her language) at 30 days and 6 months. Eight thromboembolic events occurred during the procedure, and one occurred 2 days after the procedure. No intraoperative rupture was observed. No bleeding or rebleeding was observed in the 6-month follow-up period.

\section{Progression of mRS Status}

Of the 48 patients presenting with unruptured aneurysms, at 30-day follow-up 44 were unchanged or improved with regard to $\mathrm{mRS}$ score from baseline, 1 patient declined from baseline (symptomatic thromboembolic event described above [mRS score of 1]), and 3 were not evaluated at 30 days (for 2 patients, the earlier protocol did not require a 30-day visit, and 1 patient was lost to followup). At 6 months, 44 patients were unchanged or improved from baseline; the patient with symptomatic thromboembolic event remained with an mRS score of 1; 2 patients died of cancer; and 1 patient was lost to follow-up.
Of the 3 patients presenting with ruptured aneurysms, 2 patients were Hunt and Hess Grade II and 1 patient was Hunt and Hess Grade III on entry to study. The Hunt and Hess Grade III patient improved from having an mRS score of 3 at 30 days to having an mRS score of 1 at 6 months. One patient who was Hunt and Hess Grade II had an mRS score of 0 at 30 days, and an mRS score of 0 at last follow-up (12 months). The second patient with Hunt and Hess Grade II had an mRS score of 1 at 30 days and was lost to follow-up at 6 months.

\section{Mortality/Morbidity at 1 and 6 Months}

There were no deaths at 1 month. Two deaths $(4.2 \%$ of patients with 6-month follow-up) were reported at 6 months due to cancer (mRS score of 6$)$. One patient $(2.0 \%)$ with a ruptured aneurysm had morbidity at 1 month (mRS score of 3) related to initial subarachnoid hemorrhage (Hunt and Hess Grade III) and had an mRS score of 1 at 6 months.

One patient $(2.0 \%)$ with an unruptured aneurysm had a thromboembolic event leading to an mRS score of 1 (see Technical Problems and Adverse Events) at 1 month and 6 months (no morbidity according to morbidity definition). One patient treated for an unruptured aneurysm had an $\mathrm{mRS}$ score of 2 preoperatively due to a previous subarachnoid hemorrhage (from another aneurysm) and remained at an mRS score of 2 at 1 month and 6 months (no morbidity related to the procedure). Finally, morbidity related to the treatment was $0.0 \%$ and that related to subarachnoid hemorrhage was $2.0 \%$.

\section{Postoperative Anatomical Results}

In the 48 patients effectively treated with WEB, immediate postoperative anatomical results were complete occlusion in 4 of 48 patients $(8.3 \%)$, neck remnant in 12 of 48 patients $(25.0 \%)$, and aneurysm remnant in 32 of 48 patients $(66.7 \%)$.

\section{Anatomical Results at 6 Months}

Anatomical follow-up was obtained in 41 patients (3 patients were not treated with WEB, 2 patients withdrew their consent before the 6-month follow-up, 2 patients died of cancer, and 3 patients had no 6-month follow-up imaging).

At 6 months, 23 patients $(56.1 \%)$ had complete occlu- 
sion, 12 (29.3\%) had a neck remnant, and 6 (14.6\%) had an aneurysm remnant (Fig. 1). Retreatment is foreseen in 3 of the 6 patients with an aneurysm remnant. Of the 6 patients with an aneurysm remnant, the aneurysm transverse diameters were $4.4,5.3,7.0,7.3,8.1$, and $11.1 \mathrm{~mm}$. Two aneurysms were located in the MCA, 1 in the BA, 2 in the ICA terminus, and 1 in the ACoA.

Success (complete occlusion or stable neck remnant, no worsening in angiographic appearance from postprocedure, and no retreatment performed or planned) was achieved in 35 of 41 patients $(85.4 \%)$ returning for the 6-month visit with imaging (per protocol). The lower 1-sided $95 \%$ confidence limit is $74.2 \%$, which far surpasses the WEB performance goal of $41.6 \%$ (exact binomial test, $\mathrm{p}<0.0001$ ). Since all of the subjects who underwent imaging at 6 months were in the intention-to-treat (ITT) group, the results above can be used in the ITT and per protocol analyses.

At 6-month follow-up, there were 39 patients treated with WEB without an adjunctive implant (2 patients with adjunctive devices exited the study prior to 6 months). Results in this subgroup were comparable to the overall results also; for patients without an adjunctive implant, the success rate was $87.2 \%$. Thus, by all analyses, the study met its primary end point for durable and adequate occlusion (complete occlusion or stable neck remnant) at 6 months.

\section{Discussion}

The current data confirm the high feasibility of WEB

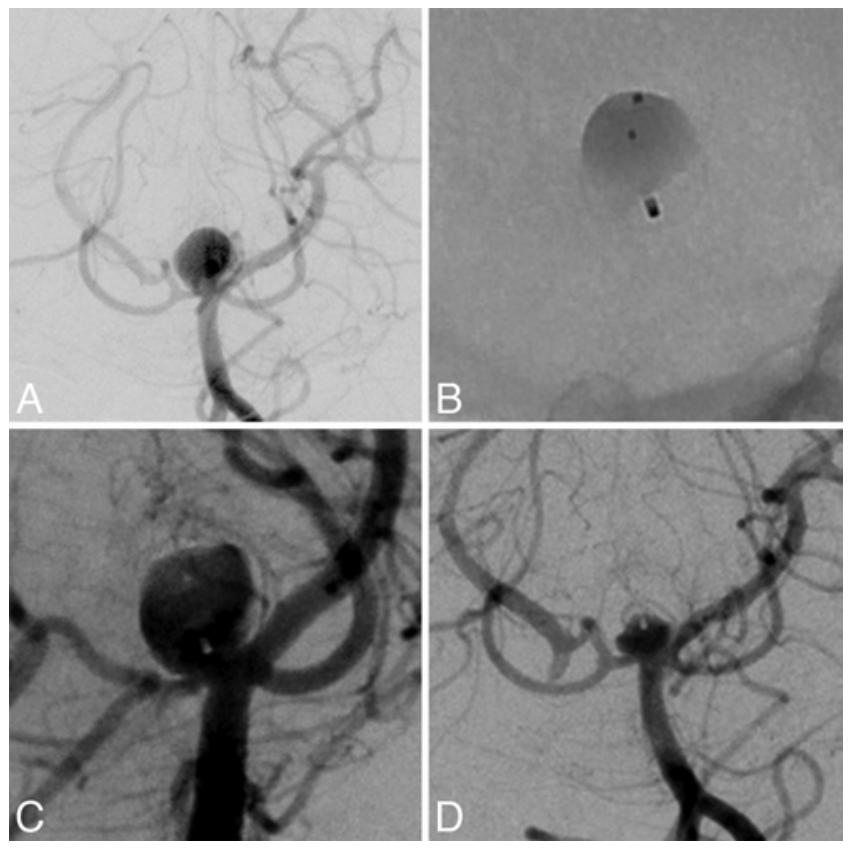

FIG. 1. A: DS angiogram showing an unruptured basilar tip aneurysm with a wide neck. B: After deployment of the WEB, the distal, intermediate, and proximal markers of the device are visible as stagnation of contrast in the device and the aneurysm. C: Immediate postdeployment DS angiogram showing the position of the device in the aneurysm with the proximal marker in the aneurysm and not in the neck (probably undersized). D: Control DS angiogram obtained at 6 months, showing the aneurysm remnant. treatment with good safety and efficacy. The limits of standard coiling have stimulated the development of new techniques for aneurysm treatment, including WEB flow

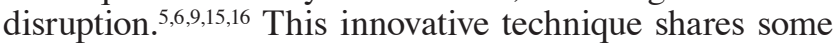
similarities and differences with both coiling and flow diversion. As with coiling, an intrasaccular device is used, but its placement in the aneurysm necessitates a larger microcatheter. Unlike coils, where several coils are typically placed in the aneurysm, in the majority of WEB cases only one device is used. WEB flow disruption shares a similar mechanism of action as flow diversion: altering the intraaneurysmal flow at the level of the neck. ${ }^{10}$ But, as WEB is intrasaccular and not intravascular, the WEB may be used for ruptured aneurysms without dual antiplatelet medication.

Preliminary WEB series have shown low morbidity rates (between $1.3 \%$ and 6.7\%), with the WEB-related mortality rate at $0.0 \%$. To confirm these data, WEBCAST was developed with high-quality methodology (external audit review of all patient files by an independent clinical monitor, independent evaluation of all adverse events, and independent analysis of anatomical results by a core laboratory).

WEBCAST was conducted during the early phase of clinical use of WEB and includes the learning curve with the WEB in terms of procedural technique, WEB sizing, and approach to antiplatelet therapy. All patients were treated with the first-generation device (WEB-DL). Data show that WEB treatment permitted the management of ruptured, unruptured, and complex wide-neck bifurcation aneurysms located at the MCA, ACoA, ICA terminus, and BA, with successful WEB deployment in 48 of 51 aneurysms (94.1\%). Despite the use of relatively large microcatheters and the first-generation device, the treatment appears to be feasible in the large majority of cases.

Most aneurysms treated in this series were unruptured $(94.1 \%)$. As this study was conducted during the initial experience for most participating centers, it is quite logical that the majority of patients treated in this series had unruptured aneurysms. However, as the WEB is an intrasaccular device, pre- and intraoperative antiplatelet medication is not mandatory. However, as shown in Table 2, 1 or 2 antiplatelet medications were given preoperatively to $58.8 \%$ of patients, intraoperatively to $76.5 \%$ of patients, and postoperatively to $82.4 \%$ of patients. Preoperative antiplatelet medication was used when treatment with a stent or flow diverter was a potential alternative to WEB treatment. The intra- and postoperative antiplatelet protocols were similar to what is currently used in coiled unruptured aneurysms. The WEB may be used for ruptured aneurysms without dual antiplatelet medication. Three ruptured aneurysms were treated in this series. This represents an important difference from aneurysm treatment techniques in which an intravascular device is used (for instance, stent and flow diverter). When these techniques are used, pre-, intra-, and postoperative dual antiplatelet treatment is mandatory, which is a significant limitation for their use, at least during the acute phase of bleeding.

The use of adjunctive devices (coils alone and/or stents) was sometimes necessary ( $8.3 \%$ of aneurysms) in an unplanned manner. Coils were used ( 3 patients) when there 
was an aneurysm remnant after WEB deployment, whereas a stent (1 patient) was used in a case of WEB device protrusion.

Overall, the safety of the endovascular treatment with WEB was high, similar to what was reported in large series dealing with ruptured or unruptured aneurysms treated with coils. The rate of thromboembolic events with WEB (17.7\%) was slightly higher than what was reported in the ATENA (unruptured aneurysms [Analysis of Treatment by Endovascular Approach of Nonruptured Aneurysms]) and CLARITY (ruptured aneurysms [Clinical and Anatomical Results in the Treatment of Ruptured Intracranial Aneurysms]) series ( $7.3 \%$ and $13.3 \%$, respectively)..$^{2,17}$ Only 1 patient $(2.0 \%)$ had a permanent deficit (mRS Score 1). It should be emphasized that the large majority of patients treated in this study had wide-neck aneurysms (92.2\% vs $30.9 \%$ in ATENA), and it is well known that the rate of thromboembolic events is higher in wide-neck aneurysms. ${ }^{11}$ There was no intraoperative rupture in this series, whereas it was observed in $2.0 \%$ of cases in ATENA and $3.7 \%$ in CLARITY. ${ }^{2,17}$

Finally, permanent morbidity at 1 month (2.0\%) was comparable to what was reported in ATENA and CLARITY (1.7\% and $3.7 \%$, respectively) and was related to the rupture status. There were no deaths. The clinical outcome was better compared with flow diverter series. In their meta-analysis of flow diversion, Arrese et al. reported the early mortality rate at $2.8 \%$ and late mortality rate at $1.3 \%{ }^{1}$ The early neurological morbidity rate was $7.3 \%$ while the late morbidity rate was $2.6 \%$.

As shown by postoperative and 6-month anatomical results, WEB treatment induces a progressive thrombosis inside the aneurysm (and the device itself) as is the case with flow diversion. It is the reason why postoperative anatomical results are mostly aneurysm remnants $(66.7 \%)$. As it is not the current clinical practice, no early imaging (during the hospital stay) was required in the protocol, and it is not possible in this trial to analyze the delay to obtain thrombosis in the device and the aneurysm.

WEBCAST shows that, in this group of complex wide-neck bifurcation aneurysms, aneurysm occlusion at 6 months is complete in 56.1\% and adequate (complete occlusion or neck remnant) in $85.4 \%$. In addition, the angiographic appearance was stable or improved compared with the end-of-treatment angiogram in all cases. Interestingly aneurysm remnants were observed in all aneurysm locations and across the range of sizes. In Table 3, these results are compared with results from a large Core Lab-evaluated series that looked at coils and stents plus coils. ${ }^{4,8,13,14,19}$ In the CLARITY trial and CCT, the rates of complete occlusion (at 16 and 6 months) were $33.8 \%$ and $28.9 \%$, respectively, whereas the rates of adequate occlusion were $81.2 \%$ and $80.9 \%$, respectively. ${ }^{8,14}$ Importantly, the success rate for the CCT, defined as complete angiographic occlusion, stable neck remnant, or improvement in angiographic appearance compared with the end-of-treatment angiogram, was $56.6 \%$ overall. For WEBCAST, this same success rate was $85.4 \%$.

As it is well known that anatomical results are worse with wide-neck aneurysms, the most realistic comparison is with the MAPS (Matrix and Platinum Science) unruptured wide-neck aneurysms subgroup, where the rate of complete occlusion (at 12 months) is $20.3 \%$ with coils and $45.7 \%$ with stenting and coiling., ${ }^{42}$ Adequate occlusion is observed in $49.1 \%$ in the coiling group and $78.6 \%$ in the stenting and coiling group. From this comparison with MAPS, WEB treatment seems to offer a higher rate of complete and adequate occlusion compared with standard coiling. If the rate of complete occlusion is higher with WEB compared with stenting plus coiling $(56.1 \%$ vs $45.7 \%$ ), the rate of adequate occlusion is relatively similar $(85.4 \%$ in WEBCAST and $78.6 \%$ in MAPS). Indeed larger, comparative studies are needed to precisely analyze efficacy of the different endovascular approaches.

This study has several limitations. First, the population is relatively small (51 patients). However, it is one of the first prospective, multicenter study evaluations with very good methodology in evaluating the safety of this treatment. Second, it is not a randomized study, and comparison with other techniques is not easy. In fact, from the study data and the literature, the safety seems to be relatively close to that of coil treatment and probably better than that of flow diverters. Compared with large series on coiling and stenting plus coiling, efficacy is at least as good and may be superior. Third, because the number of ruptured aneurysms treated in this series is low, it is difficult to precisely evaluate the place of this treatment in their management. Larger series are necessary to answer this question.

TABLE 3. Midterm anatomical results in large series

\begin{tabular}{|c|c|c|c|c|c|c|}
\hline Series & $\begin{array}{l}\text { Aneurysms Followed/ } \\
\text { Treated (no.) }\end{array}$ & $\begin{array}{l}\text { Aneurysm } \\
\text { Status }\end{array}$ & Delay & $\begin{array}{l}\text { Complete } \\
\text { Occlusion }\end{array}$ & Neck Remnant & $\begin{array}{l}\text { Aneurysm } \\
\text { Remnant }\end{array}$ \\
\hline CLARITY'13 & $517 / 649$ & $\mathrm{R}$ & Mean 16.1 mos & $33.8 \%$ & $47.4 \%$ & $18.8 \%$ \\
\hline $\mathrm{CCT}^{8}$ & $433 / 500$ & UnR/R & $\begin{array}{l}\text { Planned 6-mo follow-up (range 5-7 } \\
\text { mos) }\end{array}$ & $28.9 \%$ & $52.0 \%$ & $19.2 \%$ \\
\hline HELPS ${ }^{19}$ & $449 / 499$ & UnR/R & Mean 17.5 mos & \multicolumn{2}{|c|}{$68.4 \%$} & $31.6 \%$ \\
\hline MAPS 4 (Global) & $294 / 361$ & UnR & Planned $12 \pm 3$ & $44.4 \% * ; 51.8 \% \dagger$ & $23.9 \%{ }^{*} ; 21.1 \% \dagger$ & $31.7 \% * ; 27.2 \% \dagger$ \\
\hline MAPS 4 (WNA) & $129 / 158$ & UnR & Planned $12 \pm 3$ & $27.1 \% * ; 45.7 \% \dagger$ & $30.5 \% * ; 17.7 \% \dagger$ & $42.4 \% * ; 37.1 \% \dagger$ \\
\hline $\begin{array}{r}\text { WEBCAST } \\
(\text { WNBA })\end{array}$ & $41 / 51$ & UnR/R & $\begin{array}{l}\text { Planned 6-mo follow-up (range 3.8-7.7 } \\
\text { mos) }\end{array}$ & $56.1 \%$ & $29.3 \%$ & $14.6 \%$ \\
\hline
\end{tabular}

$\mathrm{R}=$ ruptured; UnR = unruptured; WNA = wide-neck aneurysms; WNBA = wide-neck bifurcation aneurysms.

* Coiling alone.

$\dagger$ Coiling plus stenting. 


\section{Conclusions}

The WEB safety profile is comparable to that observed with standard coiling, with very low morbidity and no study-related mortality. The WEB demonstrated a high rate of adequate occlusion (85.4\%) at 6 months, meeting the study's primary end point. This study, with independent analysis of clinical and anatomical results, confirms the good safety profile and 6-month efficacy of WEB treatment for wide-neck bifurcation aneurysms.

\section{Acknowledgment}

Dr. Byrne states that he received clinical or research support for the study described from Oxford University.

\section{References}

1. Arrese I, Sarabia R, Pintado R, Delgado-Rodriguez M: Flow-diverter devices for intracranial aneurysms: systematic review and meta-analysis. Neurosurgery 73:193-200, 2013

2. Cognard C, Pierot L, Anxionnat R, Ricolfi F : Results of embolization used as the first treatment choice in a consecutive nonselected population of ruptured aneurysms: clinical results of the Clarity GDC study. Neurosurgery 69:837-842, 2011

3. Fiorella D, Arthur A, Byrne J, Pierot L, Molyneux A, Duckwiler $\mathrm{G}$, et al: Interobserver variability in the assessment of aneurysm occlusion with the WEB aneurysm embolization system. J Neurointerv Surg [epub ahead of print], 2014

4. Hetts SW, Turk A, English JD, Dowd CF, Mocco J, Prestigiacomo C, et al: Stent-assisted coiling versus coiling alone in unruptured intracranial aneurysms in the Matrix and Platinum Science trial: safety, efficacy, and mid-term outcomes. AJNR Am J Neuroradiol 35:698-705, 2014

5. Lubicz B, Klisch J, Gauvrit JY, Szikora I, Leonardi M, Liebig T, et al: WEB-DL endovascular treatment of wide-neck bifurcation aneurysms: short- and midterm results in a European study. AJNR Am J Neuroradiol 35:432-438, 2014

6. Lubicz B, Mine B, Collignon L, Brisbois D, Duckwiler G, Strother C: WEB device for endovascular treatment of wideneck bifurcation aneurysms. AJNR Am J Neuroradiol 34:1209-1214, 2013

7. Molyneux A, Kerr R, Stratton I, Sandercock P, Clarke M, Shrimpton J, et al: International Subarachnoid Aneurysm Trial (ISAT) of neurosurgical clipping versus endovascular coiling in 2143 patients with ruptured intracranial aneurysms: a randomised trial. Lancet 360:1267-1274, 2002

8. Molyneux AJ, Clarke A, Sneade M, Mehta Z, Coley S, Roy D, et al: Cerecyte Coil Trial: angiographic outcomes of a prospective randomized trial comparing endovascular coiling of cerebral aneurysms with either cerecyte or bare platinum coils. Stroke 43:2544-2550, 2012

9. Papagiannaki C, Spelle L, Januel AC, Benaissa A, Gauvrit JY, Costalat V, et al: WEB intrasaccular flow disruptorprospective, multicenter experience in 83 patients with 85 aneurysms. AJNR Am J Neuroradiol 35:2006-2011, 2014

10. Pierot L: Flow diverter stents in the treatment of intracranial aneurysms: Where are we? J Neuroradiol 38:40-46, 2011

11. Pierot L, Cognard C, Anxionnat R, Ricolfi F: Ruptured intracranial aneurysms: factors affecting the rate and outcome of endovascular treatment complications in a series of 782 patients (CLARITY study). Radiology 256:916-923, 2010

12. Pierot L, Cognard C, Anxionnat R, Ricolfi F: Endovascular treatment of ruptured intracranial aneurysms: factors af- fecting midterm quality anatomic results: analysis in a multicenter, prospective series of patients (CLARITY). AJNR Am J Neuroradiol 33:1475-1480, 2012

13. Pierot L, Cognard C, Ricolfi F, Anxionnat R: Immediate anatomic results after the endovascular treatment of ruptured intracranial aneurysms: analysis in the CLARITY series. AJNR Am J Neuroradiol 31:907-911, 2010

14. Pierot L, Cognard C, Ricolfi F, Anxionnat R: Mid-term anatomical results after endovascular treatment of ruptured intracranial aneurysms with Guglielmi detachable coils and Matrix coils: analysis of the CLARITY series. AJNR Am J Neuroradiol 33:469-473, 2012

15. Pierot L, Klisch J, Cognard C, Szikora I, Mine B, Kadziolka $\mathrm{K}$, et al: Endovascular WEB flow disruption in middle cerebral artery aneurysms: preliminary feasibility, clinical, and anatomical results in a multicenter study. Neurosurgery 73:27-35, 2013

16. Pierot L, Liebig T, Sychra V, Kadziolka K, Dorn F, Strasilla $\mathrm{C}$, et al: Intrasaccular flow-disruption treatment of intracranial aneurysms: preliminary results of a multicenter clinical study. AJNR Am J Neuroradiol 33:1232-1238, 2012

17. Pierot L, Spelle L, Vitry F: Immediate clinical outcome of patients harboring unruptured intracranial aneurysms treated by endovascular approach: results of the ATENA study. Stroke 39:2497-2504, 2008

18. Pierot L, Wakhloo AK: Endovascular treatment of intracranial aneurysms: current status. Stroke 44:2046-2054, 2013

19. White PM, Lewis SC, Gholkar A, Sellar RJ, Nahser H, Cognard C, et al: Hydrogel-coated coils versus bare platinum coils for the endovascular treatment of intracranial aneurysms (HELPS): a randomised controlled trial. Lancet 377:1655-1662, 2011

\section{Disclosure}

The authors report that Drs. Pierot, Szikora, Klisch, Herbreteau, Holtmannspötter, Liebig, and Cognard, Molyneux, and Spelle have proctoring/consulting contracts with Sequent and that Dr. Costalat received a preclinical research grant from Sequent. The authors also report the following. Moret: consultant for Covidien, MicroVention, and Stryker; Szikora: consultant for Covidien, Codman Neurovascular, and Stryker Neurovascular; Holtmannspötter: consultant and proctor for MicroVention and Covidien; Cognard: consultant for Covidien, MicroVention, Stryker, and Codman; and Spelle, consultant for Stryker, Covidien, and Microvention.

\section{Author Contributions}

Conception and design: Pierot. Acquisition of data: Pierot, Costalat, Moret, Szikora, Klisch, Herbreteau, Holtmannspötter, Weber, Januel, Liebig, Sychra, Strasilla, Cognard, Bonafé, Molyneux, Spelle. Analysis and interpretation of data: Pierot, Molyneux, Byrne. Drafting the article: Pierot. Reviewed submitted version of manuscript: all authors. Approved the final version of the manuscript on behalf of all authors: Pierot.

\section{Supplemental Information \\ Current Affiliation}

Drs. Moret and Spelle: Assistance Publique, Hôpitaux de Paris, Bicêtre Hospital, NEURI, Le Kremlin Bicêtre, France.

\section{Correspondence}

Laurent Pierot, Department of Neuroradiology, Hôpital MaisonBlanche, 45, rue Cognacq-Jay, 51092 Reims cedex, France. email: lpierot@gmail.com. 